\title{
Programming and Compiling Web Services in GPSL
}

\author{
Dominic Cooney, Marlon Dumas, and Paul Roe \\ Queensland University of Technology, Australia \\ $\{$ d.cooney, m.dumas, p.roe\}@qut.edu.au
}

\begin{abstract}
Implementing web services that participate in long-running, multi-lateral conversations is difficult because traditional programming languages are poor at manipulating XML data and handling concurrent and interrelated interactions. We have designed a programming language to deliberately address these problems. In this paper we describe how to use this language to consume a popular web service, and discuss the compiler, including the kinds of semantic checks it performs, and the runtime environment.
\end{abstract}

\section{Introduction}

Web services are used increasingly to integrate applications within and between organizations. Implementing simple request-response interactions between statically known participants using traditional middleware and programming languages is reasonably straightforward, but implementing long-running conversations among large and changing sets of participants is difficult. Web services present some serious implementation challenges: prevalent XML data, explicit boundaries, concurrent messages, and process awareness.

$X M L$ data: The data model of web services is XML InfoSet. InfoSet is an open data representation with no notion of behavior. Object-oriented programming prizes data encapsulation by marrying data and behavior. To address the mismatch object-oriented (OO) programming languages variously model InfoSet with objects, map between objects and InfoSet, or support InfoSet directly via language extensions. In all these solutions object models are indirect, mappings are incomplete, and language extensions are redundant in their OO data model. Explicit boundaries: Unlike components in a virtual machine, or processes in an operating system, there is no supervising infrastructure between services. Because implementation technologies vary, or because organizational boundaries entail secrecy, the internal logic of other services may be completely opaque. Programming languages with global models of interacting services are useful for abstractly modeling service oriented architectures, but implementers are limited to purely local phenomena, such as messages, and can not rely on a global view. Concurrent messages: Messages link distributed nodes, all processing concurrently. For basic scalability web services must handle concurrent messages. Implementers must be cautious of race conditions, deadlocks, and live-locks_all problems that mainstream object-oriented languages make tedious to solve. 
Process awareness: Web services often correspond to business functionality, and so are likely to be part of long-running interactions driven by explicit process models. They may engage in conversations with a dynamically changing set of partners and a large number of events that may occur in many orders.

BPEL 1 addresses some of these problems, but it turns out that coding complex multi-lateral interactions in BPEL, especially those that require partial synchronization and one-to-many correlation can be cumbersome 1].

We addressed the above issues in the design of Gardens Point Service Language (GPSL) [2] with the following features:

- Embedded XQuery. XQuery is a functional language for querying and synthesizing XML data [3, with a data model close to XML InfoSet. GPSL supports the manipulation of XML data via embedded XQuery expressions.

- Services, contracts, and explicit message sending. GPSL has explicit service and contract language elements. Lexical scoping ensures services rely on purely local data. Services exchange data by explicit message sending.

- Join calculus-style concurrency [4]. GPSL simplifies forking, joining, and concurrent operations with declarative rules. At a low level of abstraction these rules facilitate the manipulation of concurrent messages; at a higher level they support the modeling of complex processes with state machines.

The feature set of GPSL is unique, yet GPSL belongs to a small set of serviceoriented programming languages [5, 6, 7]. In this paper we focus on writing service consumers, which is important for implementing services that aggregate other services. We also present the compiler and runtime system.

\section{GPSL by Example}

GPSL is primarily for developing services, and an important aspect of implementing a service is interacting with other services. In this example we describe how to use GPSL to consume the Amazon queue service. The Amazon queue service is a SOAP document/literal style service that supports inserting XML data into a queue; reading from a queue, with time-outs; and managing queues.

First we declare an XQuery XML namespace for data used by the service:

declare namespace sqs $=$

'http://webservices . amazon. com/AWSSimpleQueueService/2005-01-01';

... where sqs is a mnemonic for simple queue service.

Next we write the service contract. The Amazon queue service uses a pattern where all operations have the same SOAP action and the behavior is controlled by the data in the body of the message, so the contract declaration is simply:

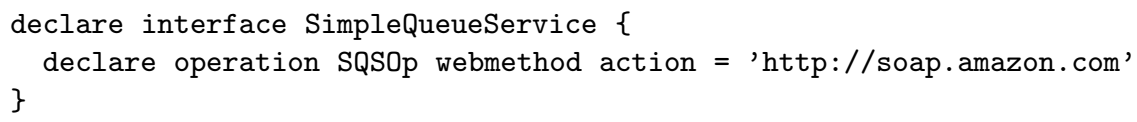

1 http://www .oasis-open.org/committees/tc_home.php?wg_abbrev=wsbpel 
SimpleQueueService and SQSOp are identifiers we use to refer to the operation. webmethod nominates this operation as synchronous SOAP-over-HTTP. This piece of metadata governs the behavior of the runtime system, but to the programmer in-out SOAP operations via a pair of asynchronous messages and synchronous webmethod operations appear uniformly as asynchronous operations.

Now we bind some constant values: the endpoint of the Amazon queue service, and our subscriber ID, which we have to include in every message. We could, of course, vary these with parameters if desired.

(: URI of the Amazon

Simple Queue Service :) let \$sqs :=

'http://webservices . amazon. com/onca/soap?Service=AWSSimpleQueueService' in

(: Amazon Web Services subscription ID :) let \$subscriptionID :=

'...' in

Performing an interaction, e.g. to create a queue, involves constructing a request, sending it, and processing the response:

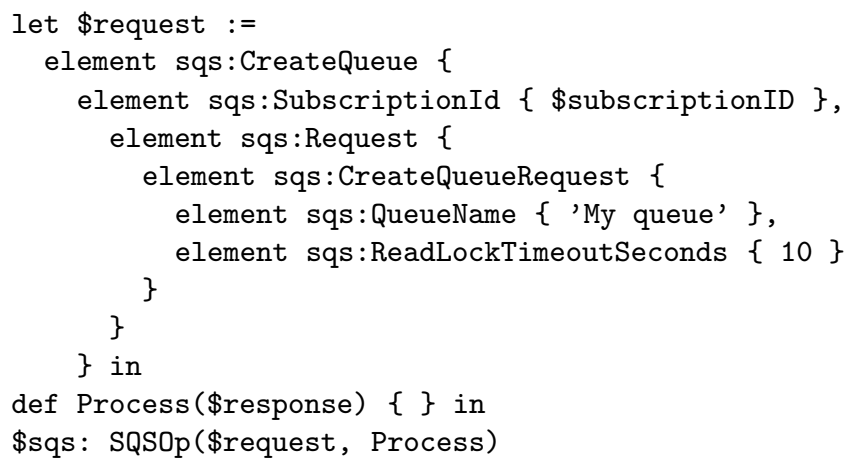

This sequence of element constructors produces XML like the following:

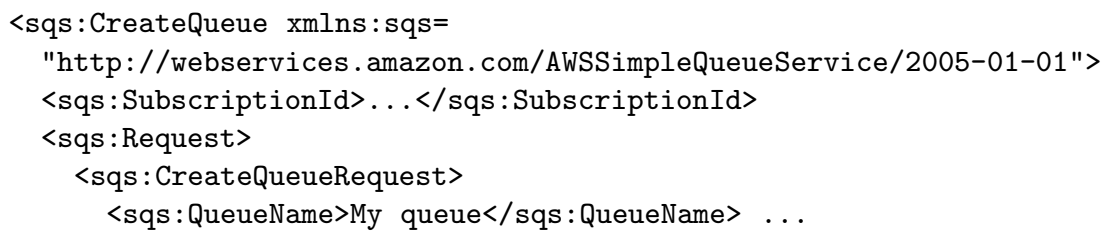

The def construct is used to introduce a new internal label, Process, and an associated block to execute when a message is produced on that label. In this example, sending a message to Process would do nothing as the block labeled Process is empty. The line \$sqs: SQSOp (\$request, Process) actually sends the message. The prefix argument $\$ s q s$ is the endpoint to send to. Recall that $\$ s q s$ is bound to the endpoint of the Amazon queue service in a previous let statement. This is how GPSL supports invoking services dynamically-for example, we could have bound $\$ s q s$, not to a constant value, but to data in a previous message.

$S Q S O p$ is the operation declared in the SimpleQueueService contract, which provides the SOAP action and operation style (webmethod, in this case) metadata. The $\$$ request argument supplies the body of the SOAP message; this is the 
fragment of XML we just constructed. Finally, the Process argument supplies the label to send SOAP replies to. Because $S Q S O p$ is declared as a webmethod, we must provide some way to handle replies.

This approach to message sending, though direct, is inconvenient if we need to create more than one queue. The GPSL def construct is very convenient for smallscale abstraction building such as the following CreateQueue label definition:

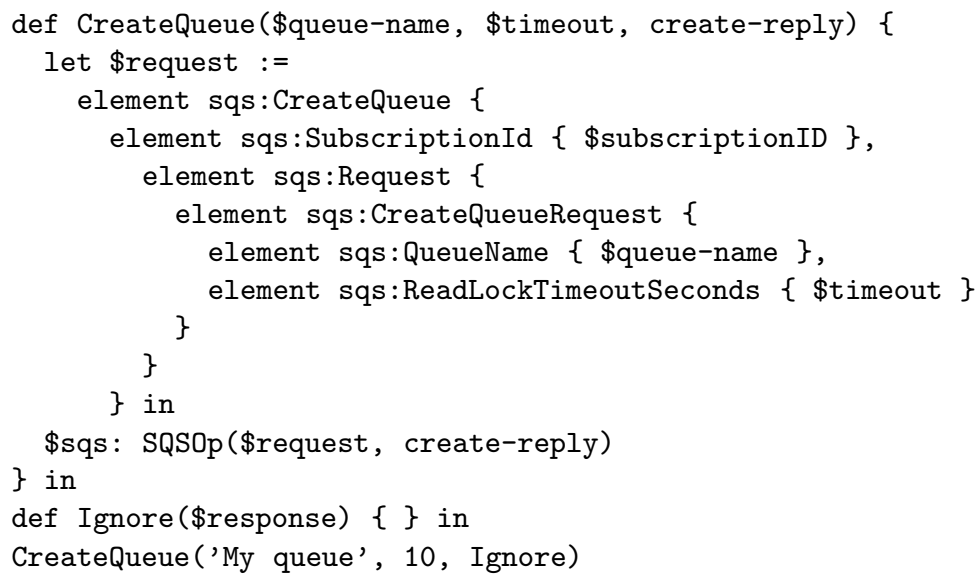

The defs statements can introduce nested defs that extract data from the response and forward the distilled result to create-reply:

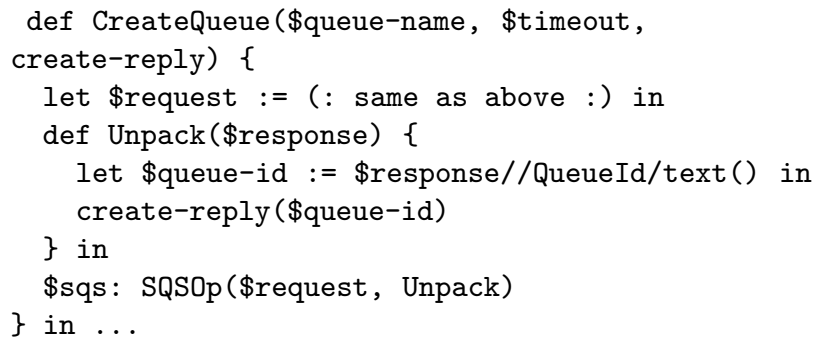

\section{The GPSL Compiler}

The GPSL compiler operates in traditional parsing, analysis, and code generation phases. The parser must handle XQuery for expressions. For our prototype we found ignoring XQuery direct constructors - the angle-brackets syntax for synthesizing XML which require special handling of whitespace-greatly simplifies parser development. Because syntactically simpler computed constructors can do the job of direct constructors, the expressive power of XQuery is unimpeded.

The analysis phase of the compiler is dominated by resolving identifiers and reporting undeclared variables or on passing too few or too many parameters. This phase includes a Hindley-Milner style type inferencer for labels. This is because we must prevent labels leaking into XML values. Syntax trivially prevents labels appearing in XQuery expressions, because variables bound to XML values 
are always prefixed with a $\$$, whereas labels and variables bound to labels are not. However sending a message on a label could pass a label where an XML value was expected. The types from our inference let the compiler guarantee statically that this does not happen.

If a label could escape into a larger XML value, we would have to track the reference to that label in order to keep the closure it refers to alive; in the worst case if the label escapes from the service we must set up the SOAP messaging machinery to marshal messages into the closure. Of course, when the programmer supplies a label as the reply-to parameter of an operation, that label is reified as an XML value. So the determined programmer can work around the restriction by sending a SOAP message to the service itself.

The last semantic check of the analysis phase is that in, in-out and webmethod operations obey the convention of a parameter for the SOAP body and a parameter for the reply channel (in the in-out and webmethod case.) These lead to a limited set of types that are used to generate code that marshal between SOAP messages and messages on internal labels. In principle, schema validation of messages could be incorporated.

The code generator produces Microsoft Intermediate Language (MSIL), which is similar to Java byte code although differs in many details. Most of the complexity in the code generator is in creating closures and delivering messages on internal labels. For each def we create a class with a method for each concurrency rule, a field for each captured variable, and a method and field for each label. This field holds a queue of pending messages; the method takes a message to that label, tests whether any rules are satisfied, and if so, calls the method for the rule. We perform the rule testing on the caller thread and only spawn a thread when a rule is satisfied, which avoids spawning many threads.

We do not compile XQuery expressions because implementing an XQuery compiler is a daunting task. Instead we generate code to call an external XQuery library at runtime. One critical criterion for the programming language implementer integrating an XQuery implementation is how that XQuery implementation accepts external variables and provides results. GPSL requires access to expression results as a sequence of XQuery data model values - which is distinctly different from an XML document - to behave consistently with XQuery when those values that are used later in subsequent expressions. We use an interoperability layer over the C API of Galax,2, which has exactly the kind of interface for providing external values and examining results that we want. Our biggest complaint about Galax is that evaluating expressions must be serialized because Galax is non-reentrant.

GPSL programs also depend on the Microsoft Web Services Extensions 3 (WSE) for SOAP messaging. WSE has a low-level messaging interface which is sufficient for GPSL's needs, but it has major shortcomings too: WSE does not support SOAP RPC/encoded, and we have to include some bookkeeping to make SOAP over synchronous-HTTP work using this low-level messaging interface.

\footnotetext{
2 http://www.galaxquery.org

3 http://msdn.microsoft.com/webservices/building/wse
} 


\section{Conclusion}

We have presented code samples in GPSL illustrating the use of features related to SOAP messaging and XQuery. Because of limited space we have not illustrated the join calculus-style concurrency features of GPSL, however the GPSL compiler and further examples are available online 4

GPSL's features for messaging, concurrency, and XML data manipulation integrate cohesively. Examples of the cohesive fit are the interplay between sending messages and spawning concurrent threads and receiving messages and synchronising threads, and the consistent treatment of inter- and intra-service messages. GPSL could be extended to address other aspects of services that are tricky to implement, such as transactions and faults. The current implementation of GPSL is also lacking in automatic resource management, expected by programmers familiar with languages such as Java or C\# but complicated by the heterogeneous, distributed setting. We expect the key in these areas is to leverage the messaging/concurrency features, for example, by surfacing faults as messages or by recovering automata from concurrency patterns and reclaiming resources when a service can no longer reach a state where it can respond to certain messages.

Acknowledgment. The second author is funded by a fellowship co-sponsored by Queensland Government and SAP.

\section{References}

1. Barros, A., Dumas, M., Hofstede, A.: Service interaction patterns. In: Proceedings of the 3rd International Conference on Business Process Management, Nancy, France, Springer Verlag (2005) Extended version available at: http://www.serviceinteraction.com.

2. Cooney, D., Dumas, M., Roe, P.: A programming language for web service development. In Estivill-Castro, V., ed.: Proceedings of the 28th Australasian Computer Science Conference, Newcastle, Australia, Australian Computer Society (2005)

3. Boag, S., Chamberlin, D., Fernández, M.F., Florescu, D., Robie, J., Siméon, J.: XQuery 1.0: An XML query language. W3C Working Draft (2005)

4. Fournet, C., Gonthier, G.: The reflexive chemical abstract machine and the join calculus. In: Twenty-third ACM Symposium on Principles of Programming Languages (POPL). (1996) 372-385

5. Onose, N., Siméon, J.: XQuery at your web service. In: Thirteenth international conference on World Wide Web, New York, NY, USA, ACM Press (2004) 603-611

6. Florescu, D., Grünhagen, A., Kossmann, D.: XL: A platform for Web services. In: Conference on Innovative Data Systems Research (CIDR), Asilomar, CA, USA (2003)

7. Kistler, T., Marais, H.: WebL - A programming language for the web. In: Proceedings of the 7th International conference on World Wide Web, Amsterdam, The Netherlands, The Netherlands, Elsevier Science Publishers B. V. (1998) 259-270

${ }^{4}$ http://www.serviceorientation.com 